\title{
STATIONARITY EQUATIONS IN CONTINUOUS TIME MARKOV CHAINS
}

\author{
BY \\ RUPERT G. MILLER, JR.(1)
}

1. Introduction. Let $\left\{X_{n}\right\}, n=0,1,2, \cdots$, be an irreducible, aperiodic Markov chain in discrete time whose state space $I$ consists of the non-negative integers. The transition probabilities are assumed to be stationary, i.e.,

$$
P\left\{X_{n+1}=j \mid X_{n}=i\right\}=p_{i j}, \quad i, j \in I, n=0,1, \cdots,
$$

where

$$
p_{i j} \geqq 0, \quad \sum_{j} p_{i j}=1, \quad i, j \in I .
$$

The matrix of transition probabilities is denoted by $P=\left(p_{i j}\right)$, and its $n$th power $P^{n}=\left(p_{i j}^{(n)}\right)$ gives the $n$-step transition probabilities. For convenience, a Markov chain satisfying these conditions will be referred to as a simple discrete time Markov chain (SDMC).

Theorem A. Let $\left\{X_{n}\right\}$ be a SDMC. A NSC for the chain to be positive recurrent is that the equations

$$
x P=x
$$

have an absolutely convergent, nonzero solution $x=\left(x_{0}, x_{1}, x_{2}, \cdots\right)$. The solution is unique except for a multiplicative constant and is

$$
x_{i}=\pi_{i}^{*}, \quad \text { where } \pi_{i}^{*}=\lim _{n \rightarrow \infty} p_{i i}^{(n)},
$$

This is a fundamental theorem on stationary distributions of Markov chains, and in applied probability, operations research, etc., the equations (1.3) are frequently solved or manipulated to obtain the limiting, stationary probabilities. The theorem is due to Foster [7] with an earlier, less general version being given by Feller [6, p. 325] (see also Chung $[1$, p. 33]).

In the null recurrent case the existence and uniqueness of a non-negative solution to the equations (1.3) without the condition of absolute convergence

Received by the editors August 28, 1962.

(1) This work was supported in part by Office of Naval Research Contract Nonr-225(52) (NR-342-022) at Stanford University. Reproduction in whole or in part is permitted for any purpose of the United States Government.

(2) The asterisk denotes a discrete time limit. 
has been established by Derman [3]. For related results in the transient case see Derman [4] and Harris [8].

Let $\left\{X_{t}\right\}, t \in T=[0, \infty)$, be an irreducible Markov chain in continuous time with the same state space $I$. The transition probability matrix $P(t)=\left(p_{i j}(t)\right)$ is assumed to be measurable and satisfy

$$
\begin{aligned}
p_{i j}(t) & \geqq 0, \quad \sum_{j} p_{i j}(t) \leqq 1, \quad i, j \in I, \\
P(t+s) & =P(t) P(s), \quad P(0+)=I,
\end{aligned}
$$

for all $t, s \in T$. In addition, the states are assumed to be stable so that

$$
\begin{array}{ll}
0>p_{i i}^{\prime}(0)=\lim _{t \downarrow 0} \frac{p_{i i}(t)-1}{t}=q_{i i}=-q_{i}>-\infty, & i \in I, \\
0<p_{i j}^{\prime}(0)=\lim _{t \downarrow 0} \frac{p_{i j}(t)}{t}=q_{i j}<+\infty, & i \neq j \in I .
\end{array}
$$

The matrix $Q=\left(q_{i j}\right)$ is called the $Q$-matrix or infinitesimal generator of the process, and it is assumed to be conservative, i.e., $\Sigma_{j} q_{i j}=0, i \in I$. For simplicity, this type of Markov chain will be referred to as a simple continuous time Markov chain (SCMC).

The equations analogous to (1.3) in continuous time are $y Q=0$. These are often referred to as the steady state or equilibrium equations (see, for example, Cox and Smith [2]) and are formally derived by setting the derivatives equal to zero in the forward Kolmogorov equations $P(t) Q=P^{\prime}(t)$ (which, perhaps unwarrantedly, are assumed to hold with equality). In applied studies these equations are used as in the discrete time process, and occasionally the following theorem is invoked.

THEOREM B. Let $\left\{X_{t}\right\}$ be a SCMC. A NSC for the chain to be positive recurrent is that the equations

$$
y Q=0
$$

have a convergent, positive solution $y=\left(y_{0}, y_{1}, y_{2}, \cdots\right)$. The solution is unique except for a multiplicative constant and is

$$
y_{i}=\pi_{i}, \quad \text { where } \pi_{i}=\lim _{t \rightarrow \infty} p_{i i}(t),
$$

$i \in I$.

Unfortunately, the theorem is not true in general.

EXAMPLE 1. Let $\left\{X_{t}\right\}$ be a birth and death process (see, for reference, Karlin and McGregor [9]). Let $q_{n, n+1}=\lambda_{n}=4^{n}, n=0,1, \cdots, q_{0,-1}=\mu_{0}=0$, and $q_{n, n-1}=\mu_{n}=4^{n} / 2, n=1,2, \cdots$. It is readily verified that $y_{n}=1 / 2^{n+1}$, $n=0,1, \cdots$, is a unique solution to $y Q=0$, but the process cannot possibly be recurrent since the probability of a birth is twice that of a death. 
In fact, Example 1 is an explosive process with $+\infty$ capable of being reached in finite time so that the process is not uniquely defined by the $\lambda_{n}$ and $\mu_{n}$. Any birth and death process for which $\mu_{0}=0$, and

$$
\sum_{n=0}^{\infty} \rho_{n}<+\infty, \quad \sum_{n=0}^{\infty} \frac{1}{\lambda_{n} \rho_{n}}<+\infty,
$$

where $\rho_{0}=1$ and $\rho_{n}=\lambda_{0} \lambda_{1} \cdots \lambda_{n-1} / \mu_{1} \cdots \mu_{n}$ would constitute a valid counterexample (see [9]).

In the next section it will be shown that Theorem B is valid provided the process is uniquely defined by the $Q$-matrix. The corresponding result in the null recurrent case is also established. In $\S 3$ the relationship between the stationary distributions of a SCMC and its imbedded discrete time Markov chain is investigated. In $\S 4$ the types of solutions to $y Q=0$ are examined for the transient and explosive processes.

2. Stationarity theorems. Unless explicitly stated to the contrary the following assumption will be in effect throughout the paper.

Assumption. The SCMC is uniquely defined by its $Q$-matrix; i.e., the minimal process is an honest process (see [1] for details).

Various necessary and sufficient conditions on the $Q$-matrix for this assumption to hold have been obtained and can be found elsewhere. For reference, see Chung [1] and Reuter [14].

TheOREM 1. Theorem B is valid (under the Assumption).

Proof. (a) Let $\left\{X_{t}\right\}$ be a positive recurrent SCMC. Then, $\pi_{i}>0, i \in I$, and $\pi=\left(\pi_{0}, \pi_{1}, \pi_{2}, \cdots\right)$ satisfies the equations

$$
\pi_{j}=\sum_{i} \pi_{i} p_{i j}(t), \quad j \in I, \quad t \in T
$$

(see $[1$, p. 179]). This can be rewritten as

$$
\pi_{j}\left(1-p_{j j}(t)\right)=\sum_{i \neq j} \pi_{i} p_{i j}(t), \quad j \in I, \quad t \in T,
$$

so by (1.6) and Fatou's lemma

$$
+\infty>\pi_{j} q_{j} \geqq \sum_{i \neq j} \pi_{i} q_{i j}>0 .
$$

(The customary trick of now summing both sides of the inequality to establish equality cannot be used because both sums may be infinite.)

The forward Kolmogorov equations, which by the Assumption hold with equality, are

$$
p_{i j}^{\prime}(t)=-p_{i j}(t) q_{j}+\sum_{k \neq j} p_{i k}(t) q_{k j}, \quad \quad i, j \in I
$$


(see [1, pp. 224-235]). Multiplication by $\pi_{i}$ and summation produces

$$
\sum_{i=0}^{N} \pi_{i} p_{i j}^{\prime}(t)=-q_{j} \sum_{i=0}^{N} \pi_{i} p_{i j}(t)+\sum_{i=0}^{N} \sum_{k \neq j} \pi_{i} p_{i k}(t) q_{k j} .
$$

The sum $\sum_{i=0}^{N} \pi_{i} p_{i j}(t)$ is non-negative, continuous in $t$, and monotonically increasing to $\pi_{j}$ as $N \rightarrow \infty$. Similarly, the second sum on the right is non-negative, continuous in $t$ (from (2.4) and [1, p. 130]), and monotonically increasing to $\sum_{i \neq j} \pi_{i} q_{i j}$ which is finite by (2.3). Hence, by Dini's theorem on uniform convergence, these sums converge uniformly for $t$ in a finite interval. Consequently, differentiation and summation can be interchanged in (2.5) when $N=+\infty$ so by (2.1)

$$
0=-\pi_{j} q_{j}+\sum_{k \neq j} \pi_{k} q_{k j}=\sum_{k} \pi_{k} q_{k j}
$$

(b) Let $y=\left(y_{0}, y_{1}, \cdots\right)$ be a convergent, positive solution to the equations $y Q=0$. Define

$$
\bar{p}_{i j}^{(n)}(t)=P\{X(t)=j, \text { and there are at most } n \text { transitions during }
$$

Then, for $i, j \in I, \quad t \in T$,

$$
(0, t) \mid X(0)=i\}
$$

$$
\begin{aligned}
& \bar{p}_{i j}^{(0)}(t)=\delta_{i j} e^{-q_{i} t} \\
& \bar{p}_{i j}^{(n)}(t)=\delta_{i j} e^{-q_{i} t}+\sum_{k \neq j} \int_{0}^{t} \bar{p}_{i k}^{(n-1)}(s) q_{k j} e^{-q_{j}(t-s)} d s,
\end{aligned}
$$

where $\delta$ is the Kronecker delta function (cf. [1, p. 228]). Clearly,

$$
\sum_{i} y_{i} \bar{p}_{i j}^{(0)}(t)=y_{j} e^{-q_{j} t} \leqq y_{j},
$$

and by induction and (1.7)

$$
\sum_{i} y_{i} \bar{p}_{i j}^{(n)}(t)=y_{j} e^{-q \jmath t}+\sum_{k \neq j} \int_{0}^{t} \sum_{i} y_{i} \bar{p}_{i k}^{(n-1)}(s) q_{k j} e^{-q_{j}(t-s)} d s
$$

$$
\leqq y_{j} e^{-q_{j} t}+\sum_{k \neq j} \int_{0}^{t} y_{k} q_{k j} e^{-q_{j}(t-s)} d s=y_{j}
$$

Since the minimal process $\bar{p}_{i j}(t)=\lim _{n \rightarrow \infty} \bar{p}_{i j}^{(n)}(t)$ coincides with $p_{i j}(t)$ by the Assumption,

$$
\lim _{n \rightarrow \infty} \sum_{i} y_{i} \bar{p}_{i j}^{(n)}(t)=\sum_{i} y_{i} p_{i j}(t) \leqq y_{j}
$$

Summation of both sides of (2.11) proves that in fact equality must hold. 
Letting $t \rightarrow \infty$ in (2.11) with equality and taking the limit inside the summation sign gives

$$
\pi_{j}=\lim _{t \rightarrow \infty} p_{i j}(t)=y_{j} / \sum_{i} y_{i}>0,
$$

which implies that the SCMC is positive recurrent and $\pi_{j}=c y_{j}$.

The first part of the proof under part (b) which utilizes the Feller construction has been used by Kendall [12] to establish some results on positive subinvariant measures. The necessity part of this theorem has been stated by Parzen [13] with a different set of conditions implied. An earlier proof of this theorem utilizing the theory of semi-groups can be found in Kendall and Reuter [11].

The statement of Theorem B cannot be weakened by replacing "convergent, positive" with "absolutely convergent, nonzero" as in the discrete case since it is possible to have signed, absolutely convergent solutions; e.g.,

EXAMPLE 2. Let

$$
\begin{aligned}
& q_{n n}=-4^{n}, \quad q_{n, n+1}=2^{n}, \quad n=0,1, \cdots, \\
& q_{10}=2, \quad q_{n, n-1}=2 \cdot 4^{n-1}-2^{n}, \quad n=2,3, \cdots, \\
& q_{n, n-2}=2 \cdot 4^{n-1}, \quad n=2,3, \cdots \text {. }
\end{aligned}
$$

Then, $y_{n}=(-1 / 2)^{n+1}, n=0,1, \cdots$, is an absolutely convergent, signed solution to $y Q=0$.

By virtue of the equivalence theorem of Smith $[15$, p. 22], Theorem B also applies to regular, aperiodic semi-Markov processes. A regular (i.e., nonexplosive) aperiodic semi-Markov process is completely defined by its transition probability matrix $P=\left(p_{i j}\right)$, which governs the successive state transitions, and its transition distribution function matrix $F=\left(F_{i j}\right)$, which determines the length of sojourn in each state. Smith's theorem proves that the limiting $(t \rightarrow \infty)$ probabilities of the process are invariant under changes in $F$ as long as $\mu=\left(\mu_{i j}\right)=\left(\int_{0}^{\infty} t d F_{i j}(t)\right)$ remains invariant. Hence, in dealing with limiting probabilities, $F$ can be assumed to be exponential as in a Markov process, and Theorem 1 can be applied with

$$
\begin{aligned}
q_{i}^{-1} & =\sum_{j} p_{i j} \mu_{i j}, & i \in I, \\
q_{i j} & =q_{i} p_{i j}, & i \neq j \in I .
\end{aligned}
$$

(The quantity $q_{i}$ is assumed to be nonzero. If $p_{i i} \neq 0$, equations (2.14) should be modified slightly.)

The analog to Derman's discrete time result holds as well.

THEOREM 2. Let $\left\{X_{t}\right\}$ be a recurrent SCMC. The equations $y Q=0$ have a unique (except for a multiplicative constant) positive solution which is 


$$
y_{i}=e_{i}^{(h, k)}={ }_{h} P_{k i}(\infty)+{ }_{k} P_{h i}(\infty), \quad i \in I, h \neq k \in I,
$$

where

$$
{ }_{h} p_{k i}(t)=P\{X(s) \neq h, 0<s<t, X(t)=i \mid X(0)=k\}
$$

$$
{ }_{h} P_{k i}(\infty)=\int_{0}^{\infty}{ }_{h} p_{k i}(t) d t
$$

(For properties of taboo probability functions the reader is referred to [1].) Proof. (a) By Theorem II.13.5 of $\left[1\right.$, p. 202] $\left({ }^{3}\right)$, for any fixed pair $(h, k)$, $h \neq k \in I$,

$$
e_{j}^{(h, k)}=\sum e_{i}^{(h, k)} p_{i j}(t), \quad j \in I, t \in T
$$

But now part (a) of the proof of Theorem 1 can be repeated verbatim with $e_{i}^{(h, k)}$ replacing $\pi_{i}$ since it nowhere utilizes the property $\Sigma_{i} \pi_{i}<+\infty$.

(b) To establish uniqueness the first part of (b) under Theorem 1 can be repeated to obtain

$$
\sum_{i} y_{i} p_{i j}(t) \leqq y_{j}, \quad j \in I, t \in T .
$$

The inversion technique of Feller-Derman with a slight modification will establish equality in (2.18). For fixed $t$ define

$$
r_{i j}^{(n)}=\frac{y_{j}}{y_{i}} p_{j i}(n t)
$$

$i, j \in I$.

The matrix $R=\left(r_{i j}^{(1)}\right)$ is a substochastic transition matrix since

$$
\begin{aligned}
r_{i j}^{(n)} & \geqq 0, \quad \sum_{j} r_{i j}^{(n)} \leqq 1, \\
\sum_{j} r_{i j} r_{j k}^{(n)} & =\sum_{j} \frac{y_{j}}{y_{i}} p_{j i}(t) \frac{y_{k}}{y_{j}} p_{k j}(n t) \\
& =\frac{y_{k}}{y_{i}} \sum_{j} p_{k j}(n t) p_{j i}(t)=r_{i k}^{(n+1)} .
\end{aligned}
$$

(3) The proof of Theorem II.13.5 is incomplete in that the sentence following expression (20) is invalid. Corrected proofs have been given by Chung and S. Orey, and at the suggestion of Chung the somewhat shorter proof of Orey is included below.

In the notation of [1] the proof is modified beginning with line 11 from the bottom: $e_{l}^{(i, j)}(t / m)$ $=\Sigma_{k} e_{k}^{(i, j)}(t / m) p_{k l}(t)$. Multiplying through by $t / m$ gives $(t / m) e_{l}^{(i, j)}(t / m)=\Sigma_{k}\left[(t / m) e_{k}^{(i, j)}(t / m)\right] p_{k l}(t)$. According to Theorem I.9.7 there exist constants $c^{(m)}$ such that for $m=1,2, \cdots,(t / m) e^{(i, j)}(t / m)$ $=c^{(m)} t e_{k}^{(i, j)}(t), k \in I$. Hence, for $k \in I, t>0,(20) e_{k}^{(i, j)}(t)=\lim _{m \rightarrow \infty}\left((t / m) e^{(i, j)}(t / m) / t c^{(m)}\right)$ $=e_{k}^{(i, j)} / t c, 0<c<\infty$, by Theorem 13.3 (in whose statement the - in $(11)$ and the entire expression (12) should be deleted). Substitution of (20) into $e_{l}^{(i, j)}(t)=\Sigma_{k} e_{k}^{(i, j)}(t) p_{k l}(t)$ completes the proof of the first part of the theorem. 
To convert $R$ into a stochastic transition matrix add an additional absorbing state $\infty$ with

$$
r_{i \infty}=1-\sum_{j} r_{i j}, \quad r_{\infty \infty}=1
$$

$i \in I$.

Since

$$
\sum_{n} r_{i i}^{(n)}=\sum_{n} p_{i i}(n t)=+\infty
$$

$i \in I$

(by $[1$, p. 180]), all states $i \in I$ are recurrent for the chain defined by $R$. But this is impossible if $r_{i \infty}>0$ for any $i$ since $\infty$ is an absorbing state. Thus, $\sum_{j} r_{i j}^{(n)}=1$ for $i \in I$, which is equivalent to equality in (2.18).

The uniqueness part of Theorem II.13.5 of [1] proves that

$$
y_{i}=e_{i}^{(h, k)}, \quad i \in I, \quad h \neq k \in I .
$$

3. Imbedded Markov chain. Every simple continuous time Markov chain has an imbedded discrete time Markov chain whose transitions consist of the successive state changes in the continuous chain whenever they occur. The elements of the transition probability matrix $P$ for the imbedded chain are $p_{i j}=q_{i j} / q_{i}$. The imbedded chain is necessarily irreducible but may conceivably be periodic (in which case $\pi_{i}^{*}$ is the Cesàro limit).

If the continuous chain is recurrent so is the imbedded chain and vice versa. However, this does not extend to the positiveness of the recurrence.

EXAMPLE 3. Let $\left\{X_{t}\right\}$ be a birth and death process with $\mu_{0}=0$ and $\lambda_{n}=\mu_{n}$, $n=1,2, \cdots$. The process is uniquely defined (by [9, pp. 527-532]) since

$$
\sum_{n=0}^{\infty} \frac{1}{\lambda_{n} \rho_{n}} \sum_{m=n}^{n} \rho_{m}=\sum_{n=0}^{\infty} \frac{1}{\lambda_{0}} \sum_{m=0}^{n} \frac{\lambda_{0}}{\mu_{m}}=+\infty
$$

$$
\sum_{n=0}^{\infty} \frac{1}{\lambda_{n} \rho_{n}}=\sum_{n=0}^{\infty} \frac{1}{\lambda_{0}}=+\infty,
$$

where $\rho_{0}=1$, and $\rho_{n}=\lambda_{0} \lambda_{1} \cdots \lambda_{n-1} / \mu_{1} \cdots \mu_{n}, n>0$. If $\mu_{n} \rightarrow+\infty$ sufficiently rapidly so that

$$
\sum_{n=0}^{\infty} \rho_{n}=\sum_{n=0}^{\infty} \frac{\lambda_{0}}{\mu_{n}}<+\infty
$$

then the process is positive recurrent (by [10, p. 370]). However, the imbedded chain cannot be positive recurrent since $p_{n, n-1}=p_{n, n+1}=1 / 2$.

EXAMPLE 4. Let $\left\{X_{n}\right\}$ be any positive recurrent SDMC with $p_{i i}=0, i \in I$. From Theorem A

$$
\pi_{j}^{*}=\sum_{i} \pi_{i}^{*} p_{i j}, \quad j \in I
$$


where $\pi_{i}^{*}>0, \Sigma_{i} \pi_{i}^{*}=1$. From (3.3)

$$
\left(\pi_{j}^{*} / q_{j}\right) q_{j}=\sum_{i \neq j}\left(\pi_{i}^{*} / q_{i}\right) q_{i} p_{i j}
$$

$j \in I$,

for any sequence of positive constants $q_{0}, q_{1}, \cdots$. Choose $q_{i}=\pi_{i}^{*}, i \in I$. Then, for the continuous chain with

$$
q_{i i}=-q_{i}, \quad q_{i j}=q_{i} p_{i j}
$$

$i, j \in I$,

the Assumption is satisfied since the $q_{i}$ are bounded (see $[1$, p. 237]), and the continuous chain is null recurrent by Theorem 2 since $\pi_{i}^{*} / q_{i}$ is the unique, positive solution to $y Q=0$ and $\sum \pi_{i}^{*} / q_{i}=+\infty$.

THEOREM 3. In a recurrent chain the unique (except for multiplicative constants) positive solutions of $x P=x$ and $y Q=0$, where $p_{i j}=q_{i j} / q_{i}, p_{i i}=0$, $i, j \in I$, are related by $x_{i}=y_{i} q_{i}, i \in I$. If $\pi_{i}^{*}>0$ and $c=\sum_{i} \pi_{i}^{*} / q_{i}<+\infty$, then $\pi_{i}=c^{-1} \pi_{i} / q_{i}>0$, and if $\pi_{i}>0$ and $c=\sum_{i} \pi_{i} q_{i}<+\infty$, then $\pi_{i}^{*}=c^{-1} \pi_{i} q_{i}>0$.

Proof. The first part of the theorem is immediate from

$$
\left(x_{j} / q_{j}\right) q_{j}=\sum_{i \neq j}\left(x_{i} / q_{i}\right) q_{i} p_{i j}
$$

$j \in I$.

The second part follows from the first part and Theorems A and B.

This theorem yields the following characterization of the invariant measure for a null recurrent SCMC: for fixed $h, k, l \in I, h \neq k$,

where

$$
e_{i}^{(h, k)}={ }_{\imath} p_{l i}^{*} / q_{i}
$$

$$
\begin{aligned}
& { }_{l} p_{l i}^{(n)}=P\left\{X_{m} \neq l, 0<m<n, \quad X_{n}=i \mid X_{0}=l\right\}, \\
& { }_{l} p_{l i}^{*}=\sum_{n=1}^{\infty}{ }_{l} p_{l i}^{(n)},
\end{aligned}
$$

and $\left\{X_{n}\right\}$ is the imbedded Markov chain. The quantity ${ }_{i} p_{l i}^{*} / q_{i}$ is the expected length of time spent in state $i$ between visits to state $l$ (which equals ${ }_{l} P_{l i}(\infty)$ defined in [1]).

Since this theorem applies as well to regular, aperiodic semi-Markov processes, it generalizes a result of Fabens [5].

4. Solution of $y Q=0$. In $\S 2$ the family of positive solutions of $y Q=0$ was shown to consist of a single member in the positive recurrent and null recurrent cases. This ceases to be true for transient and explosive processes where the families can also be vacuous or contain a multiplicity of members. Various possibilities for the different cases are summarized below. It is to be understood that only positive solutions are under consideration, and unless stated to the contrary the solution is nonconvergent. 
1. Positive recurrent: unique, convergent solution.

2. Null recurrent: unique solution.

3. Transient: if Derman's [4] examples of a renewal type chain (with $\left.p_{0}=1\right)$ and asymmetrical unrestricted random walk are adapted to continuous time by letting all the exponential sojourn distributions have the same parameter, they constitute, respectively, examples of

(a) no solution,

(b) nonunique solution.

For the simplest example of

(c) unique solution,

any birth and death process with $\mu_{0}=0$,

$$
\sum_{n=0}^{\infty} \frac{1}{\lambda_{n} \rho_{n}}<+\infty, \quad \sum_{n=0}^{\infty} \rho_{n}=+\infty, \quad \sum_{n=0}^{\infty} \frac{1}{\lambda_{n} \rho_{n}} \sum_{m=0}^{n} \rho_{m}=+\infty
$$

will suffice (see [10, pp. 376-377]).

The Assumption is dropped for the next case.

4. Explosive. (a) No solution.

Derman's renewal type chain $\left(p_{0}=1\right)$ with any set of sojourn parameters $q_{i}, i \in I$, for which $\sum_{i} q_{i}^{-1}<+\infty$ is an example.

(b) Nonunique solution.

An asymmetrical random walk with invariant transition probabilities $(q, p)$ and variable sojourn parameters $q_{i}$ (i.e., a bilateral birth and death process; $I=\{\cdots,-1,0,1, \cdots\})$ has the solutions

$$
y_{i}=\frac{1}{q_{i}}\left(c_{1}\left(\frac{p}{q}\right)^{i}+c_{2}\right), \quad i=0, \pm 1, \pm 2, \cdots,
$$

where $c_{1}, c_{2}>0$. Let $p>q$, and choose $q_{i} \equiv q>0, i=-1,-2, \cdots$, and $q_{i}=(p / q)^{2 i}, i=0,1, \cdots$. Then, all three series in (4.1) are convergent so the process is explosive, but (4.2) is nonunique since $c_{1}, c_{2}>0$ are arbitrary.

(c) Unique solution.

Any birth and death process with $\mu_{0}=0$,

$$
\sum_{n=0}^{\infty} \frac{1}{\lambda_{n} \rho_{n}}<+\infty, \quad \sum_{n=0}^{\infty} \rho_{n}=+\infty, \quad \sum_{n=0}^{\infty} \frac{1}{\lambda_{n} \rho_{n}} \sum_{m=0}^{n} \rho_{m}<+\infty,
$$

is an example.

(d) Unique, convergent solution.

See Example 1.

(e) Nonunique, convergent solution.

In (4.2) select $q_{i}=(p / q)^{2|i|}, i=0, \pm 1, \cdots$. 


\section{BIBLIOGRAPHY}

1. K. L. Chung, Markov chains with stationary transition probabilities, Springer-Verlag, Berlin, 1960.

2. D. R. Cox and W. L. Smith, Queues, Methuen, London, 1961.

3. C. Derman, $A$ solution to a set of fundamental equations in Markov chains, Proc. Amer. Math. Soc. 5 (1954), 332-334.

4. - Some contributions to the theory of denumerable Markov chains, Trans. Amer. Math. Soc. 79 (1955), 541-555.

5. A. J. Fabens, The solution of queueing and inventory models by semi-Markov processes J. Roy Statist. Soc. Se1. B 23 (1961), 113-127.

6. W. Feller, An introduction to probability theory and its applications, Wiley, New York, 1950.

7. F. G. Foster, On the stochastic matrices associated with certain queueing processes, Ann. Math. Statist. 24 (1953), 355-360.

8. T. E. Harris, Transient Markov chains with stationary measures, Proc. Amer. Math. Soc. 8 (1957), 937-942.

9. S. Karlin and J. McGregor, The differential equations of birth-and-death processes, and the Stieltjes moment problem, Trans. Amer. Math. Soc. 85 (1957), 489-546.

10. - - The classification of birth and death processes, Trans. Amer. Math. Soc. 86 (1957), 366-400.

11. D. G. Kendall and G. E. H. Reuter, The calculation of the ergodic projection for Markov chains and processes with a countable infinity of states, Acta Math. 97 (1957), 103-144.

12. D. G. Kendall, Unitary dilations of one-parameter semigroups of Markov transition operators, and the corresponding integral representations for Markov processes with a countable infinity of states, Proc. London Math. Soc. 9 (1959), 417-431.

13. E. Parzen, Stochastic processes, Holden-Day, San Francisco, Calif., 1962.

14. G. E. H. Reuter, Denumerable Markov processes and the associated contraction semigroups on l, Acta Math. 97 (1957), 1-46.

15. W. L. Smith, Regenerative stochastic processes, Proc. Roy. Soc. Ser. A 232 (1955), 6-31.

STANFORD UNIVERSITY,

StANFord, CALIFORNia 